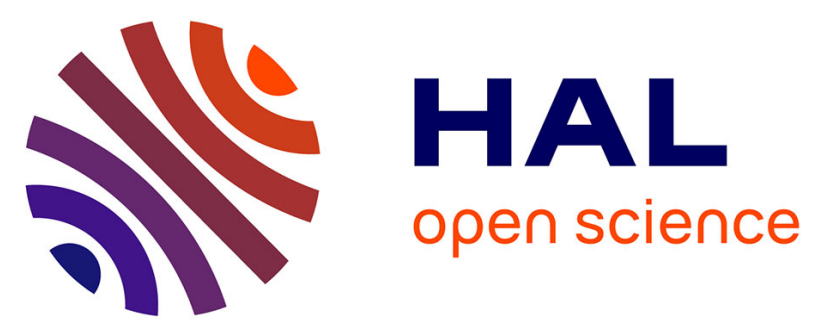

\title{
Dark solitons, dispersive waves and their collision in an optical fiber
}

T. Marest, C. Mas Mas Arabí, Matteo Conforti, Arnaud Mussot, C. Milian, D. Skryabin, Alexandre Kudlinski

\section{- To cite this version:}

T. Marest, C. Mas Mas Arabí, Matteo Conforti, Arnaud Mussot, C. Milian, et al.. Dark solitons, dispersive waves and their collision in an optical fiber. Nonlinear Photonics, Jul 2018, Zurich, France. NpW4C.3， 10.1364/NP.2018.NpW4C.3 . hal-02393083

\section{HAL Id: hal-02393083 \\ https://hal.science/hal-02393083}

Submitted on 4 Dec 2019

HAL is a multi-disciplinary open access archive for the deposit and dissemination of scientific research documents, whether they are published or not. The documents may come from teaching and research institutions in France or abroad, or from public or private research centers.
L'archive ouverte pluridisciplinaire HAL, est destinée au dépôt et à la diffusion de documents scientifiques de niveau recherche, publiés ou non, émanant des établissements d'enseignement et de recherche français ou étrangers, des laboratoires publics ou privés. 


\title{
Dark solitons, dispersive waves and their collision in an optical fiber
}

\author{
T. Marest ${ }^{1}$, C. Mas Arabí ${ }^{1}$, M. Conforti ${ }^{1}$, A. Mussot ${ }^{1}$, C. Milian ${ }^{2}$, D. V. Skryabin ${ }^{2,3}$ and A. Kudlinski ${ }^{1}$ \\ ${ }^{1}$ Univ. Lille, CNRS, UMR 8523 - PhLAM - Physique des Lasers Atomes et Molécules, F-59000 Lille, France \\ ${ }^{2}$ Department of Physics, University of Bath, Bath BA2 $7 A Y$, United Kingdom \\ ${ }^{3}$ ITMO University, St. Petersburg 197101, Russian Federation \\ alexandre.kudlinski@univ-lille.fr
}

\begin{abstract}
We report the experimental observation of dispersive wave emission from gray solitons in an optical fiber. We also observed the nonlinear wave mixing occurring during the collision of a dark soliton and a linear wave.

OCIS codes: (190.4370) Nonlinear optics, fibers; (190.5530) Pulse propagation and temporal solitons.
\end{abstract}

Dark solitons are a class of solution of the defocusing nonlinear Schrödinger equation (NLSE) [1]. In optical fibers, this corresponds to the normal dispersion region. They exhibit an intensity dip (associated with a phase jump) over a uniform background. Dark solitons having a zero intensity at their core and an abrupt $\pi$ phase jump are called black solitons or fundamental dark solitons. When the minimum intensity does not drop to zero and the phase change is smooth and smaller than $\pi$, they are called gray solitons. The amplitude of dark solitons take the form

$$
A(t)=\cos \varphi \tanh \left(\frac{t}{T_{0}} \cos \varphi\right)-i \sin \varphi
$$

where $\varphi$ is the grayness parameter ( $\varphi=0$ corresponds to the black soliton) and $T_{0}$ is the duration of the dark dip. In this work, we observe experimentally for the first time the process of dispersive wave emission from dark solitons, in accordance with old theoretical predictions [2,3], and we study the impact of dark soliton grayness on the efficiency of dispersive wave emission. Finally, we observe the nonlinear wave mixing occurring during the collision between a dark soliton and a dispersive wave.

We generate the required odd dark pulses by using two commercially available waveshapers. Short pulses from an optical parametric oscillator $(220 \mathrm{fs}, 1550 \mathrm{~nm})$ are sent to a first waveshaper in which only the amplitude is shaped. At this stage, the grayness of the dark pulses can be controlled accurately. Then, the generated dark pulses are amplified and sent to a second waveshaper where the antisymmetric phase profile is applied. The resulting pulses are shaped both in amplitude and phase and have therefore the required properties to excite a dark solitons in the fiber (see example of cross-correlation in Fig. 1a and spectrum in Fig. 1b measurements of the shaped pulses at the fiber input). These pulses are then launched in a $3.15 \mathrm{~km}$ long dispersion shifted fiber (DSF), in the low normal dispersion region. Figure 1c shows the measurement of the output spectrum for various values of the grayness parameter $\varphi$. For $\varphi$ values between $-\pi / 6$ and $\pi / 20$, a dispersive wave is emitted across the zero-dispersion wavelength (ZDW) and follows the theoretical phase-matching curve (black solid line), while for $\varphi$ values outside this range, none is observed. In Fig. 1d, we plot the energy of dispersive wave as a function of grayness parameter. The theoretical curve (red line)
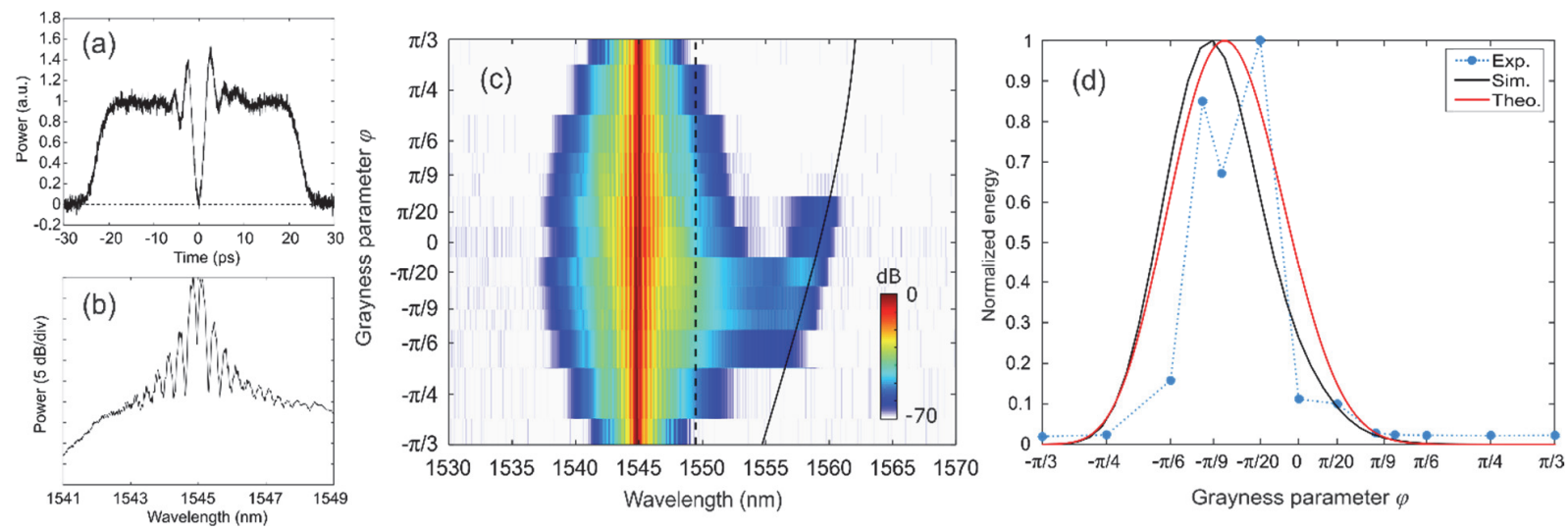

Fig. 1 : (a)-(b) Example of temporal and spectral measurement of the input odd dark pulse. (c) Measured spectra at the fiber output as a function of grayness parameter (black solid line: phase-matching relation, black dashed line: ZDW). (d) Dispersive wave energy as a function of grayness parameter (black line: generalized NLSE simulations, red line : theory adapted from [4], blue markers: measurements). 
is obtained by adapting the theory developed for bright solitons in [4] to the dark soliton case. Black line is obtained from numerical simulations of the generalized NLSE and markers correspond to measurements, in good agreement. For large $|\varphi|$ values, the dark soliton spectrum is narrow, so that the spectral overlap with anomalous dispersion region is low, which explain the decrease in dispersive wave emission efficiency (similarly to the bright soliton case [5]). Additionally, for large positive $\varphi$ values, the theoretical phase-matching wavelength away from the ZDW, which further reduces the dispersive wave efficiency.

In a second set of experiments, we study the collision of a dispersive wave with a dark soliton. The experimental setup is mainly the same as in the previous experiment, but instead of being generated from the dark soliton in the fiber, here the dispersive wave is shaped directly in the input short pulse spectrum. The first waveshaper used to shape the dark pulse and the dispersive wave pulse (called the probe here) at a different wavelength, and the second waveshaper is used to adjust the odd-symmetry phase profile of the dark pulse and the delay of the probe pulse.
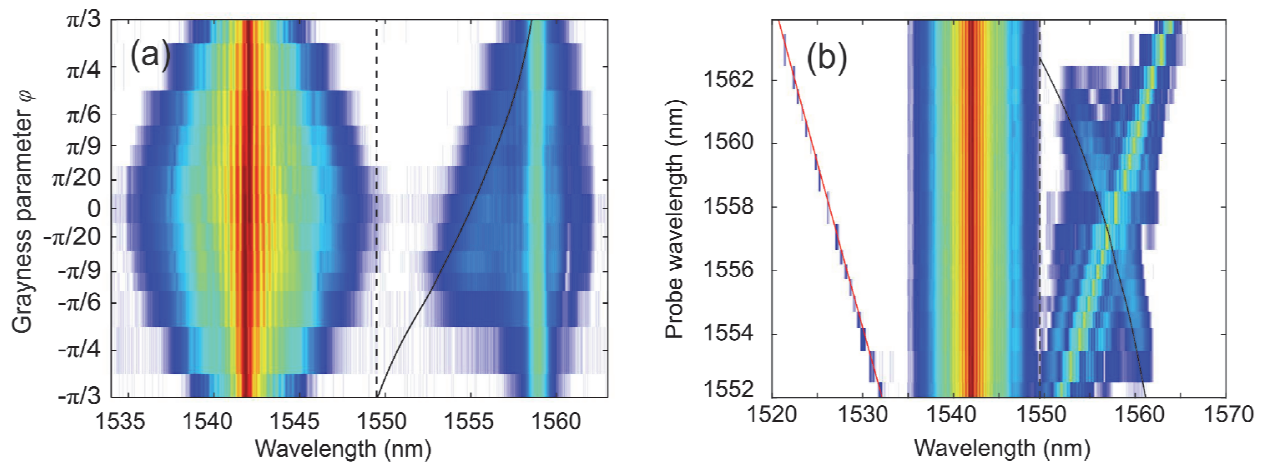

Fig. 2 : Experimental observation of the nonlinear wave mixing occurring during the collision between a dark soliton and a dispersive wave. (a) Measurement of the output spectrum as a function of (a) dark soliton grayness for a fixed probe wavelength of $1559 \mathrm{~nm}$ and (b) probe wavelength for a fixed soliton grayness $\varphi=\pi / 10$. Dashed line: ZDW, black solid line: theoretical solution of FWM between the dark soliton and the probe, red solid line: theoretical of FWM between the dark soliton background and the probe.

Figure $2 \mathrm{a}$ shows the measurement of the output spectrum for various values of the grayness parameter. The dark soliton is centered around $1542 \mathrm{~nm}$ and the probe wave is located at $1559 \mathrm{~nm}$. For $\varphi$ values higher than $-\pi / 6$, a radiation resulting from the four wave mixing (FWM) between the probe and the dark soliton is observed [6]. It closely follows the FWM phase-matching relation (black solid line) that we have obtained by adapting the well-known theory for bright solitons [7]. In Fig. 2b, we show the measured spectra obtained by varying the probe wavelength, for a gray soliton with $\varphi=\pi / 10$. Again, a new spectral component resulting from the FWM occurring at the collision between the dark soliton and the probe wave is observed, in excellent agreement with the theoretical curve (black solid line). In this case, we also observe a new radiation at short wavelength, that we can identify as the FWM between the probe wave and the quasi-cw background over which the dark soliton is located (red solid line). Numerical simulations of the generalized NLSE (not shown here) are in excellent agreement with the experiments of Fig. 2a and b. Additionally, we can derive analytically the conversion efficiency of the FWM process, again by adapting the bright soliton theory from e.g. [8], and we can simulate it using the generalized NLSE. The results (not shown here) are in excellent agreement with the experiments of Figs. $2 \mathrm{a}$ and $\mathrm{b}$. A detailed analysis of these results will be presented at the conference.

To summarize, we have observed a grayness-dependent emission of dispersive waves from dark solitons in an optical fiber. We have also observed a grayness-dependent generation of new radiations resulting from the four-wave mixing process occurring during the collision of dark solitons and linear waves.

\section{References}

1. Y. S. Kivshar and B. Luther-Davies, "Dark optical solitons: physics and applications," Phys. Rep. 298, 81-197 (1998).

2. V. I. Karpman, "Stationary and radiating dark solitons of the third order nonlinear Schrödinger equation," Phys. Lett. A 181, 211-215 (1993).

3. V. V. Afanasjev, Y. S. Kivshar, and C. R. Menyuk, "Effect of third-order dispersion on dark solitons," Opt. Lett. 21, 1975-1977 (1996).

4. F. Biancalana, D. V. Skryabin, and A. V. Yulin, "Theory of the soliton self-frequency shift compensation by the resonant radiation in photonic crystal fibers," Phys. Rev. E 70, 016615 (2004).

5. N. Akhmediev and M. Karlsson, "Cherenkov radiation emitted by solitons in optical fibers," Phys. Rev. A 51, 2602-2607 (1995).

6. I. Oreshnikov, R. Driben, and A. V. Yulin, "Weak and strong interactions between dark solitons and dispersive waves," Opt. Lett. 40, 4871$4874(2015)$

7. A. V. Yulin, D. V. Skryabin, and P. S. J. Russell, "Four-wave mixing of linear waves and solitons in fibers with higher-order dispersion," Opt. Lett. 29, 2411-2413 (2004).

8. A. Choudhary and F. König, "Efficient frequency shifting of dispersive waves at solitons," Opt. Express 20, 5538-5546 (2012). 